\title{
Systematic review of the dry powder inhalers colistimethate sodium and tobramycin in cystic fibrosis
}

\author{
Lesley Uttley', Sue Harnan', Anna Cantrell', Chris Taylor², Martin Walshaw³, \\ Keith Brownlee ${ }^{4}$ and Paul Tappenden ${ }^{1}$
}

\begin{abstract}
Affiliations: ${ }^{1}$ School of Health and Related Research (ScHARR), University of Sheffield, Sheffield, ${ }^{2}$ Department of Gastroenterological and Hepatological Medicine, Sheffield Children's Hospital, Sheffield Children's NHS Foundation Trust, Sheffield, ${ }^{3}$ Adult Cystic Fibrosis Unit, Liverpool Heart and Chest Hospital NHS Foundation Trust, Liverpool, and 'Paediatric and Adult Regional Cystic Fibrosis Centre, Leeds Teaching Hospitals NHS Trust, Leeds, UK.
\end{abstract}

Correspondence: L. Uttley, School of Health and Related Research (ScHARR), University of Sheffield, Regent Court, 30 Regent Street, Sheffield S1 4DA, UK. E-mail: l.uttleydasheffield.ac.uk

ABSTRACT This systematic review evaluated evidence for two dry powder formulations, colistimethate sodium and tobramycin, for the treatment of chronic Pseudomonas aeruginosa in cystic fibrosis, as part of the UK national recommendation process for new technologies. Electronic bibliographic databases were searched in May 2012 (MEDLINE, MEDLINE in-Process, EMBASE, Cochrane Library databases, CINAHL, Web of Science, Conference Proceedings Citation Index and BIOSIS Previews). Relevant outcomes included rate and extent of microbial response (e.g. sputum density of $P$. aeruginosa), lung function (e.g. forced expiratory volume in $1 \mathrm{~s}(\mathrm{FEV} 1)$ ), frequency, severity of acute exacerbations and adverse events. Three trials were included, and both dry powder formulations were reported to be non-inferior in the short term to nebulised tobramycin for FEV1. However, long-term follow-up data were missing and the effect on exacerbation rates was not always reported. Whilst short-term results showed that both dry powder drugs were non-inferior to nebulised tobramycin, there was no long-term follow-up and no phase 3 trials compared nebulised and dry powder colistimethate sodium. The use of FEV1 as the primary end-point may not accurately represent changes in lung health. This review illustrates the difficulty in assessing new technologies where the evidence base is poor.

0 @ERSpublications

It is difficult to assess potential benefits of new, innovative technologies when the evidence base for efficacy is poor http://ow.ly/nVbCP

\section{Introduction}

Pseudomonas aeruginosa is the most prevalent lung infection in people with cystic fibrosis (CF) [1], and the majority of adults in the UK are chronically infected [2]. It is associated with increased mortality and morbidity [3]. Current practice is to treat incidences of chronic infection using oral or nebulised antibiotics (or both, depending on the UK centre) $[4,5]$ with the aim of suppressing the infection.

Received: March 192013 | Accepted after revision: April 262013

Support statement: This article was funded as part of project number 10/08/01 for the National Institute for Health Research, Health Technology Assessment Programme and the National Institute for Health and Care Excellence (NICE). The views expressed in this document are not necessarily those of NICE.

Conflict of interest: Disclosures can be found alongside the online version of this article at err.ersjournals.com

Provenance: Submitted article, peer reviewed.

ERR articles are open access and distributed under the terms of the Creative Commons Attribution Non-Commercial Licence 3.0. 
Both colistimethate sodium and tobramycin in nebulised form are well established in the CF community for this purpose. However, nebulising medication involves preparation of the drug, which can be time consuming and requires the use of expensive nebuliser apparatus that requires maintenance. Both these factors increase the treatment burden and may reduce compliance and, therefore, treatment efficacy.

Recently, new dry powder formulations of colistimethate sodium (Colobreathe; Forest Laboratories, Dartford, UK) and tobramycin (TOBI Podhaler; Novartis Pharmaceuticals, Camberley, UK) have become available, delivered by convenient portable mechanisms (Turbospin device (Forest Laboratories) and T-326 Inhaler (Novartis Pharmaceuticals)). One of the principal anticipated benefits of dry powder antibiotics is that they are quicker to use and are more portable than traditional nebulised therapy [6]. It is hypothesised that these benefits could reduce the burden of treatment for many CF patients and may lead to improvements in adherence. Such improvements could theoretically result in increased efficacy of the antipseudomonal antibiotic treatments and result in improvements to quality of life. In order to assess this potential benefit, adherence to the study drug should be measured in addition to the clinical efficacy of the drugs and overall quality of life using a preference based health-related quality of life (HRQoL) instrument. Whilst dry powder delivery of antibiotics may be a welcome innovation in CF treatment, the reliability and efficacy of such devices are yet to be universally established for effective drug delivery [7].

The objective of this systematic review was to inform the National Institute for Health and Clinical Excellence (NICE), London, UK, about the clinical effectiveness and cost-effectiveness of two dry powder inhaler (DPI) formulations of anti-pseudomonal antibiotics for chronic P. aeruginosa infection in patients with CF. This review includes assessment of two interventions that are delivered as a DPI: colistimethate sodium DPI (Colobreathe used with the Turbospin delivery device) and tobramycin DPI (TOBI Podhaler used with the Podhaler delivery device). The antibiotics colistin and tobramycin also represent the relevant comparators for the assessment, albeit in nebulised form. Both interventions are intended for the ongoing treatment of chronic $P$. aeruginosa.

\section{Methods}

The scope of the review was determined by NICE for the assessment of the clinical effectiveness and costeffectiveness of the two newly developed drug formulations, each in combination with their respective patented devices [8].

\section{Identification of studies}

A comprehensive search was undertaken to systematically identify randomised controlled trials relating to the clinical effectiveness of colistimethate sodium DPI and tobramycin DPI for the treatment of $P$. aeruginosa in CF. The broader evidence network for a mixed treatment comparison was also examined, but was not included in this review as a network could not be constructed due to clinical heterogeneity between trials; for more details see [9]. The following electronic databases were searched for published trials and systematic reviews: MEDLINE, EMBASE, Cochrane Library, CINAHL, Web of Science Citation Index, Conference Proceedings Citation Index, and BIOSIS Previews. Ongoing and unpublished studies were searched for in relevant databases. Drug manufacturer submissions from Forest Laboratories UK Ltd and Novartis Pharmaceuticals were received by NICE. Any relevant systematic reviews identified during the review process were also checked in order to identify any further clinical trials. No date or language restrictions were applied. Literature searches were initially conducted in March 2011 and replicated and updated in May 2012.

\section{Populations and subgroups}

The population for the assessment included people aged $\geqslant 6$ years with CF and chronic P. aeruginosa pulmonary colonisation.

\section{Interventions}

Two interventions were included in this systematic review: 1) colistimethate sodium DPI used in conjunction with the Turbospin device; and 2) tobramycin DPI used in conjunction with the Podhaler device.

\section{Relevant comparators}

The interventions are compared against each other. Other relevant comparators include antipseudomonal antibiotics used for nebulised inhalation, including colistimethate sodium and tobramycin for nebulised inhalation.

\section{Outcomes}

The outcomes considered within this review were rate and extent of microbial response (e.g. sputum density of $P$. aeruginosa), lung function, for example measurements in terms of forced expiratory volume in 
$1 \mathrm{~s}$ (FEV1) [10], respiratory symptoms, frequency and severity of acute exacerbations, HRQoL and adverse events of treatment (including rate of resistance to antibiotic treatment).

\section{Exclusion criteria}

The following were excluded: studies based on animal models; preclinical and biological studies; nonrandomised controlled trials; editorials and opinion pieces; reports published as meeting abstracts only, where insufficient details were reported to allow inclusion; and studies in which the population was not restricted to $\mathrm{CF}$.

\section{Study selection and evidence synthesis}

Studies that satisfied the inclusion and exclusion criteria were selected for inclusion in the review. Risk of bias was assessed using the Centre for Reviews and Dissemination criteria [11]. Studies were assessed as high, low or unclear risk for items including participant recruitment, randomisation, blinding and study reporting. Data extraction was conducted using a standardised data extraction form that had been piloted and adapted to include all relevant data fields. Study selection, data extraction and quality assessment were completed by one reviewer and checked by a second reviewer. Discrepancies were discussed and resolved with a third party. A narrative synthesis was conducted. A meta-analysis was planned subject to the availability of suitable data.

\section{Results}

\section{Quantity of research available}

The search retrieved 866 potentially relevant citations; 857 from searches of electronic databases, and nine from secondary searches of relevant reviews, articles and the sponsor's submissions. Of these, 841 were excluded at the title and abstract stage, leaving 25 potentially includable citations.

The full texts of the 25 articles were obtained for investigation; of these, 20 did not meet the inclusion criteria and were excluded (fig. 1)[12]. Three studies comparing colistimethate sodium DPI or tobramycin DPI with a nebulised antibiotic were included in the review. One study (the EAGER trial) investigated tobramycin DPI in combination with the TOBI Podhaler, and two studies (COLO/DPI/02/06 and COLO/ DPI/02/05) investigated colistimethate sodium DPI in combination with the Turbospin device. Information about the three trials included in the systematic review was initially available from five sources; these comprised one published journal article [13], two conference abstracts [14, 15] and two manufacturers' submissions to NICE $[16,17]$ (with subsequent clarifications from the manufacturers). Additional sources became available during the appraisal process comprising two European Public Assessment Reports (EPARs), which were published by the European Medicines Agency (EMA) (for TOBI Podhaler in 2011, updated in 2012 [18], and for Colobreathe in 2012 [1]), and a journal article relating to the COLO/DPI/02/06 study [19]. Whilst EPAR sources are not peer reviewed, they are scrutinised through the EMA licensing process. Data received after seeking clarifications from both manufacturers, but which had not been made publicly available, are not reported here.

\section{Study characteristics}

All studies were open label, multicentre studies, two of which were multinational studies. A summary of characteristics of the included studies can be found in table 1 . The EAGER trial $[13,16,18]$ was a large trial $(n=533)$ performed between 2006 and 2009 that compared tobramycin DPI and nebulised tobramycin. The COLO/DPI/02/06 trial $[1,17]$ was a slightly smaller trial $(n=380)$ that finished data collection in 2007 and compared colistimethate sodium DPI with nebulised tobramycin. Both of these trials were powered to detect clinically relevant changes in FEV1. The COLO/DPI/02/05 trial was much smaller $(n=16)$, and the dates of the study were not reported; compared colistimethate sodium DPI with nebulised colistimethate sodium. The EAGER trial and COLO/DPI/02/06 studies were both 24 weeks in duration, whilst COLO/ DPI/02/05 was a crossover trial that reported outcome data at 4 weeks (before crossover) and 8 weeks only (after crossover).

\section{Inclusion and exclusion}

Criteria were largely comparable between the two major trials (EAGER and COLO/DPI/02/06). Patients in all three trials had a chronic $P$. aeruginosa infection and were clinically stable. They were aged $>6$ years in the EAGER and COLO/DPI/02/06 trials, and $>8$ years in the COLO/DPI/02/05 trial. Patients in all trials continued with usual CF treatments (except other routine anti-pseudomonal treatments). The criteria used to define a chronic infection did not meet with EMA recommendations in any trial [10]. As such, it is the comparability of the degree of infection that is unclear between the included patients in the trials. 


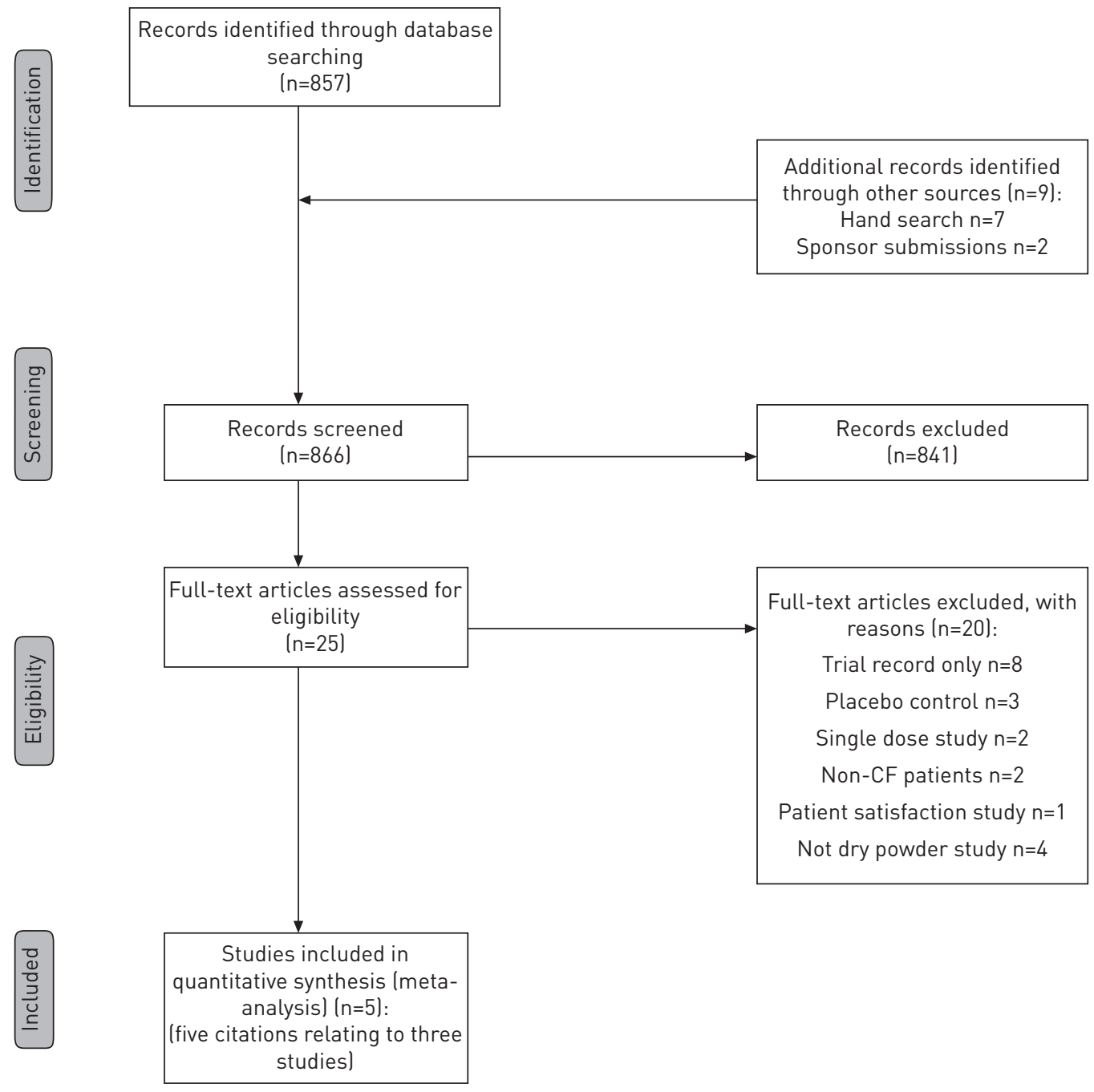

FIGURE 1 Flow diagram of study inclusion. CF: cystic fibrosis.

The COLO/DPI/02/06 trial had a run-in period whereby participants were required to have received 16 weeks (two cycles) of nebulised tobramycin prior to beginning the trial in order to eliminate the shortterm peak in FEV1 \% predicted efficacy, which has been documented in patients receiving tobramycin, and which is not sustained over time [20]. Conversely, the EAGER trial had a proportion of patients (6.2\%) who had not used tobramycin or any other anti-pseudomonal antibiotic before, and a substantial proportion $(67.6 \%)$ who had not used anti-pseudomonal antibiotics immediately prior to the 28-day wash-out period. Some or all of these patients may have experienced an initial peak in efficacy during the trial, and may also be more likely to experience adverse events associated with tobramycin than patients in the COLO/DPI/02/06 trial.

\section{Patient characteristics}

Baseline characteristics of patients are presented in table 2. The patients in the COLO/DPI/02/06 trial had a lower mean age than those in the EAGER trial. Mean age was not reported for the COLO/DPI/02/05 trial. The baseline FEV1 \% pred values were similar between these two trials, with the FEV1\% pred in COLO/ DPI/02/06 being slightly lower, indicating that patients had slightly worse lung health to start with overall in comparison to the patients in the EAGER trial. In both trials, the lack of consistency in inclusion criteria and conformity with EMA guidelines [10] affects the comparability of the trials to one another.

\section{Study withdrawals}

In both trials, more patients withdrew due to adverse events than for any other single reason. Withdrawal of consent/patient request was the second most common reason. In the EAGER trial, adverse events accounted for proportionately more withdrawals in the tobramycin DPI arm (13\%) than in the nebulised tobramycin $\operatorname{arm}(8.1 \%)$. Similarly, more patients withdrew consent from the trial in the DPI arm (8\% versus $4.3 \%)$. 
TABLE 1 Summary of included studies

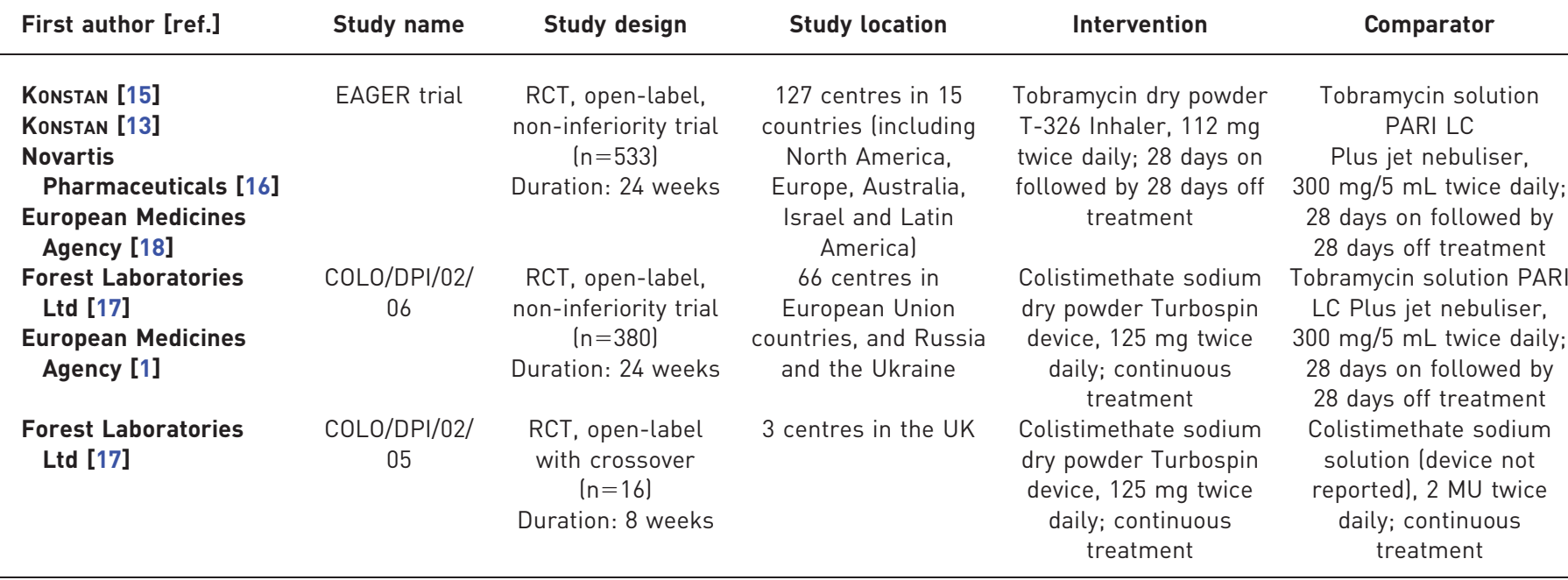

$\mathrm{RCT}$ : randomised controlled trial.

In the COLO/DPI/02/06 trial, the same pattern was seen with more patients withdrawing from the colistimethate sodium DPI arm $(9.8 \%)$ than from the nebulised tobramycin arm $(1.6 \%)$ due to adverse events, although withdrawals due to patient request were lower in the DPI arm (2.7\% versus $5.8 \%)$.

\section{Quality assessment}

All three trials randomised patients to treatment. The method of randomisation was acceptable in the EAGER trial, but was not clearly described in the COLO/DPI/02/06 trial. The EAGER and COLO/DPI/02/06 studies adopted a method of allocation concealment using an interactive voice system. Participants were not blinded to the treatment arm in two of the studies (EAGER and COLO/DPI/02/06), although the Forest Laboratories UK Ltd submission states that FEV1 data were collected by a blinded investigator. The COLO/ DPI/02/05 study does not state whether any blinding was attempted. Due to the fact that part of the assessment was to establish patient convenience, blinding would have been inappropriate. However, this still represents a risk of bias for potential benefit and harm from both patients and investigators.

Two of the studies (COLO/DPI/02/06 and COLO/DPI/02/05) reported that relatively similar numbers of patients in the intervention and control groups dropped out of the study; however, data did not support this. The EAGER trial reported a somewhat higher attrition in the intervention group (26.9\%) compared to the control group $(18.2 \%)$ and, as data were not corrected for withdrawals, the study may therefore be at high risk of attrition bias. It would seem that more evidence was recorded than was reported for some FEV1 $\%$ pred measurements and for body mass index (BMI) in the EAGER trial, which may indicate a degree of reporting bias. In addition, there was a substantial delay in publishing the trial results for the COLO/DPI/ 02/06 study, which finished collecting data in 2007. The results have recently been published in a peerreviewed journal [19]. Delayed publication of clinical trial data is an area of concern for reporting the results of empirical work generally, but is a specific concern for trials in CF [21].

\section{Assessment of effectiveness \\ Lung function}

The most commonly reported measure of lung function from the included studies was FEV1. Where available, data were obtained at 4, 20 and 24 weeks. Whilst outcome data for FEV1 at 24 weeks were provided in the data submissions from Forest Laboratories UK Ltd, only data at 20 weeks was available in full from any source for the EAGER trial. Tobramycin was administered for 28 days followed by 28 days off treatment, which results in a peak and trough in FEV1 values. This has the potential to affect estimates of comparative efficacy, and it would seem appropriate to consider results at both the peak and trough of the efficacy cycle. As such, data from both 20 and 24 weeks are presented within this review where possible.

The presentation and analysis of data varied across the studies. The COLO/DPI/02/06 reported several analyses for FEV1 data, including intention-to-treat (ITT) and per protocol populations with last observation carried forward (LOCF) imputation and without imputation. Tests specified a priori showed that the data were non-normal in distribution; therefore, additional non-parametric analyses and analyses 
TABLE 2 Baseline characteristics of patients

\begin{tabular}{|c|c|c|c|c|c|c|c|c|}
\hline \multirow[t]{2}{*}{ Study } & \multicolumn{2}{|c|}{ Age years } & \multicolumn{2}{|c|}{ Males } & \multicolumn{2}{|c|}{ BMI } & \multicolumn{2}{|c|}{ FEV1 \% pred } \\
\hline & Int. & Comp. & Int. & Comp. & Int. & Comp. & Int. & Comp. \\
\hline $\begin{array}{l}\text { COLO/DPI/02/ } \\
06\end{array}$ & $21.3 \pm 9.72$ & $20.9 \pm 9.30$ & 103/183 (56.3) & 101/190 (52.9) & $18.67 \pm 3.396$ & $18.46 \pm 3.584$ & $\begin{array}{c}\text { Mean } \pm \text { SE } \\
51.76 \pm 1.02\end{array}$ & $\begin{array}{c}\text { Mean } \pm \text { SE } \\
50.82 \pm 0.99\end{array}$ \\
\hline $\begin{array}{l}\text { COLO/DPI/02/ } \\
05\end{array}$ & \multicolumn{2}{|c|}{$\begin{array}{c}\geqslant 8 \text { to }<13 \text { years } 37.5 \% \\
\geqslant 13 \text { years } 62.5 \%\end{array}$} & NR & NR & \multicolumn{2}{|c|}{$19.99 \pm 4.011$} & $\begin{array}{c}\text { Mean } \pm \text { SE } \\
75.92 \pm 11.86\end{array}$ & $\begin{array}{c}\text { Mean } \pm \text { SE } \\
79.51 \pm 7.707\end{array}$ \\
\hline
\end{tabular}

Data are presented as mean \pm SD or $\mathrm{n} / \mathrm{N}(\%)$, unless otherwise stated. BMI: body mass index; FEV1: forced expiratory volume in $1 \mathrm{~s} ; \%$ pred: $\%$ predicted; Int.: intervention; Comp.: comparator; NR: not reported.

using logarithmically transformed data were also performed by the manufacturer to correct for this. The EAGER trial data in the manufacturer's submission were not transformed, and a non-parametric test was not performed, although no test of normality was apparently planned or performed. No imputation was performed on the Novartis data, and only limited data were presented at 24 weeks. Some adjusted comparative data were presented, with adjustments for main effects treatment, baseline FEV1 \% pred and pooled centre.

Non-inferiority was defined in the COLO/DPI/02/06 trial as having been satisfied if the lower limit of the $95 \%$ confidence interval was no lower than $-3.0 \%$ for both the per protocol and ITT populations. It was also stated that the primary efficacy analysis was performed using a LOCF imputation and that the Shapiro-Wilk test would be used to check for non-normal distribution. Non-inferiority was reported in the study by SCHUSTER et al. [12] for logarithmic analysis (table 3) but not for the original analysis on the absolute scale. Additional data from the industry submission indicates that the conclusion on non-inferiority in the logarithmic analysis is based upon patients with data at baseline and 24 weeks (completers), in the per protocol population. However, the LOCF analysis did not demonstrate non-inferiority. A further nonparametric analysis was conducted as tests indicated that the assumption of normality was not fully satisfied by the logarithmic transformation and non-inferiority was demonstrated in this analysis. Similar results were reported for data at 20 weeks.

Non-inferiority was defined in the EAGER trial as having been satisfied if the lower limit of the 95\% confidence interval was no lower than $-6 \%$ in the ITT population. No imputation was carried out and no tests for normality were planned. Data were available at 20 weeks but not 24 weeks. The EAGER trial reported non-inferiority for tobramycin DPI at 20 weeks, supported by least squares mean \pm SE difference relative change of $1.1 \pm 1.75 \%$, which meets the criteria for non-inferiority. As noted previously, this analysis was performed with no imputation of data in the ITT population. A non-inferiority analysis was not presented for the data at 24 weeks, where FEV1\% measurements are expected to be lower for both study arms than at 20 weeks. It is unclear whether this would have affected the estimate of comparative efficacy between the two arms.

Both the EAGER trial and the COLO/DPI/02/06 trial conclude that tobramycin DPI and colistimethate sodium DPI, respectively, are non-inferior to nebulised tobramycin as they have met their respective predefined non-inferiority criteria. However, it is unclear whether the standards for non-inferiority are comparable between trials.

\section{Microbiological outcomes: colony density and resistance}

In the COLO/DPI/02/06 trial, resistance $\left(4 \mathrm{mg} \cdot \mathrm{L}^{-1}\right.$ or $8 \mathrm{mg} \cdot \mathrm{L}^{-1}$ breakpoints) to colistimethate sodium remained very low $(\leqslant 1.1 \%)$ in the colistimethate sodium DPI arm, whilst resistance to tobramycin did not change substantially during the study. In the EAGER trial, resistance $\left(8 \mathrm{mg} \cdot \mathrm{L}^{-1}\right.$ breakpoint $)$ to tobramycin started at around $20 \%$ and was lower at 24 weeks. It is unclear if this change was statistically significant.

Sputum density tests were conducted in the EAGER trial only. Mean change from baseline $\log _{10}$ values showed numerically greater reductions in sputum density and were achieved with tobramycin DPI at 20 weeks in comparison to nebulised tobramycin. Results at 24 weeks were not reported. Statistical significance was not reported.

\section{Exacerbations}

Data on protocol defined acute exacerbations were not reported in a consistent way across the three included trials. The EAGER trial did not report acute exacerbations and did not have a protocol definition 
TABLE 3 Forced expiratory volume in $1 \mathrm{~s}$ (FEV1) estimates of efficacy for the COLO/DPI/02/06 trial

\begin{tabular}{|c|c|c|c|c|c|c|c|c|}
\hline Analysis & Population & $\begin{array}{l}\text { Data included } \\
\text { in analysis }\end{array}$ & CDPI & TIS & $\begin{array}{l}\text { Adjusted mean } \\
\text { difference between } \\
\text { groups in FEV1 } \% \\
\text { from baseline }\end{array}$ & $\begin{array}{l}\text { Lower limit } \\
\text { of } 95 \% \mathrm{Cl}^{\oplus}\end{array}$ & $\begin{array}{l}\text { Upper limit } \\
\text { of } 95 \% \mathrm{Cl}\end{array}$ & $\begin{array}{c}\text { Satisfies } \\
\text { non-inferiority? }\end{array}$ \\
\hline
\end{tabular}

\begin{tabular}{|c|c|c|c|c|c|c|c|c|}
\hline on the original & & Completers & 153 & 171 & -0.43 & -2.59 & 1.72 & Yes \\
\hline \multirow{2}{*}{ absolute scale } & PP & LOCF & 141 & 157 & -1.49 & -3.79 & 0.81 & No \\
\hline & & Completers & 120 & 141 & -0.99 & -3.48 & 1.51 & No \\
\hline \multirow{3}{*}{$\begin{array}{l}\text { Logarithmic } \\
\text { analysis }\end{array}$} & & Completers & 153 & 171 & -0.29 & -2.20 & 1.70 & Yes \\
\hline & $\mathrm{PP}$ & LOCF & 141 & 157 & -1.10 & -3.08 & 0.97 & No \\
\hline & & Completers & 120 & 141 & -0.56 & -2.71 & 1.70 & Yes \\
\hline \multirow{2}{*}{$\begin{array}{l}\text { Non-parametric } \\
\text { analysis }\end{array}$} & PP & LOCF & 141 & 157 & $-0.67^{+}$ & $-2.57^{+}$ & NR & Yes \\
\hline & & Completers & 120 & 141 & $-0.15^{+}$ & $-2.14^{+}$ & NR & Yes \\
\hline
\end{tabular}

Data are presented as $\mathrm{n}$ or \%. Bold denotes protocol-defined analyses. CDPI: colistimethate sodium dry powder for inhalation; TIS: tobramycin inhaled solution; ITT: intention-to-treat; PP: per protocol; LOCF: last observation carried forward; NR: not reported. \#: adjusted for baseline $\mathrm{FEV} 1 \%$ and pooled centre; ${ }^{\text {: }}$ : this should no lower than $-3 \%$ to satisfy non-inferiority criteria; ${ }^{+}$: median difference, $\mathrm{Cl}$ determined using distribution-free methods based on the Wilcoxon rank sum test, without adjustment for pooled centre; unclear if adjusted for baseline FEV1 \%.

of an exacerbation. Instead they report "lung disorders" defined as "generally reported by the investigator as pulmonary or CF exacerbation" [13]. This is not an entirely specific measure and it is unclear what other events may also have been included in this outcome. However, the percentage of patients experiencing at least one episode of lung disorder was 33.8\% (dry powder) versus 30.1\% (nebulised).

The COLO/DPI/02/06 trial did report protocol defined acute exacerbations. Numerically, more patients on colistimethate sodium DPI experienced acute exacerbations compared to nebulised tobramycin in COLO/ DPI/02/06 (31.1\% versus $26.1 \%$, respectively), although it is unclear if the same trend would be observed for data relating to the number of events. Acute exacerbations were not reported in the COLO/DPI/02/05 trial. It is not possible to draw a comparative conclusion as to the relative efficacy between trials in terms of exacerbations, given the difference in the way they have been reported, and the uncertainty about the comparability of the patient data populations and characteristics.

\section{Body mass index}

The EAGER trial reported baseline BMI but did not report data for time-points at the end of the trial at either 20 or 24 weeks [16]. The COLO/DPI/02/06 trial reported BMI data for the ITT population, which demonstrated very little change in BMI from baseline [17]. BMI data for the COLO/DPI/02/05 was not presented as it was not an outcome under investigation in that trial.

\section{Health-related quality of life}

Two trials investigated this outcome (COLO/DPI/02/06 and COLO/DPI/02/05). COLO/DPI/02/06 data are presented as the adjusted means of Cystic Fibrosis Questionnaire (CFQ) domains from baseline to week 24. Most of the scores tended to be in favour of the dry powder intervention although none of the differences are statistically significant. This quality of life measure has not been validated and is not preference based. The COLO/DPI/02/05 trial also used the CFQ measure but the data are not available in the public domain. There was no quality of life data reported in the EAGER trial. However, treatment satisfaction was measured using the Treatment Satisfaction Questionnaire for Medication.

\section{Adverse events}

The percentage of patients experiencing any adverse event was high in the EAGER trial (DPI 90.3\% versus nebulised 84.2\%), the COLO/DPI/02/06 trial (DPI 93.6\% versus nebulised 89.1\%) and the COLO/DPI/02/ 05 trial (nebulised 100\% versus DPI 60\%), although this is to be expected in a patient population with CF who have a high level of baseline adverse events. The EAGER trial did not state how many events were severe, whilst both of the colistimethate sodium DPI trials reported more severe events in the intervention (DPI 39.0\%) arm versus the control (nebulised 6.2\%) arm. This can, in part, be explained by the fact that 
TABLE 4 Most common adverse events ( $\geqslant 5 \%$ in any group) across the three studies

EAGER

\begin{tabular}{|c|c|c|c|c|c|c|}
\hline & $\begin{array}{c}\text { Tobramycin } \\
\text { DPI }\end{array}$ & $\begin{array}{l}\text { Nebulised } \\
\text { tobramycin }\end{array}$ & $\begin{array}{l}\text { Colistimethate } \\
\text { sodium DPI }\end{array}$ & $\begin{array}{l}\text { Nebulised } \\
\text { tobramycin }\end{array}$ & $\begin{array}{l}\text { Colistimethate } \\
\text { sodium DPI }\end{array}$ & $\begin{array}{l}\text { Nebulised } \\
\text { colistin }\end{array}$ \\
\hline Patients $\#$ & 308 & 209 & 187 & 193 & 16 & 15 \\
\hline Throat irritation & $\mathrm{AiC}$ & $\mathrm{AiC}$ & $141(75.4)$ & $84(43.5)$ & 13 (81.3) & $3(20.0)$ \\
\hline Productive cough & $56(18.2)$ & $41(19.6)$ & 38 (20.3) & $44(22.8)$ & $2(12.5)$ & $1(6.7)$ \\
\hline Dyspnoea & $48(15.6)$ & $26(12.4)$ & $49(26.2)$ & 52 (26.9) & $3(18.8)$ & $4(26.7)$ \\
\hline Rhinorrhoea & $22(7.1)$ & $15(7.2)$ & $1(0.5)$ & $2(1.0)$ & NR & NR \\
\hline $\begin{array}{l}\text { Pulmonary function } \\
\text { test decreased }\end{array}$ & $21(6.8)$ & $17(8.1)$ & $0(0)$ & $3(1.6)$ & NR & NR \\
\hline Pyrexia & $48(15.6)$ & $26(12.4)$ & $23(12.3)$ & $19(9.8)$ & 2 (12.5) & $1(6.7)$ \\
\hline Dysgeusia & $\mathrm{AiC}$ & $\mathrm{AiC}$ & $117(62.6)$ & 53 (27.5) & 14 (87.5) & $3(20.0)$ \\
\hline Respiratory disorders & $21(6.8)$ & $18(8.6)$ & $53(28.3)$ & 57 (29.5) & $16(100)$ & $7(46.7)$ \\
\hline Dysphonia & $42(13.6)$ & $8(3.8)$ & $22(11.8)$ & $30(15.5)$ & NR & NR \\
\hline Nasal congestion & $25(8.1)$ & $15(7.2)$ & $2(1.1)$ & $4(2.1)$ & NR & NR \\
\hline Vomiting & $19(6.2)$ & $12(5.7)$ & 6 (3.2) & $8(4.1)$ & $2(12.0)$ & $0(0)$ \\
\hline Haemoptysis & $40(13.0)$ & $26(12.4)$ & $20(10.7)$ & $13(6.7)$ & NR & NR \\
\hline Nausea & $23(7.5)$ & $20(9.6)$ & $7(3.7)$ & $9(4.7)$ & NR & NR \\
\hline Headache & $35(11.4)$ & $25(12.0)$ & $9(4.8)$ & $16(8.3)$ & $1(6.3)$ & $2(13.3)$ \\
\hline Fatigue & $20(6.5)$ & $10(4.8)$ & $9(4.8)$ & $8(4.1)$ & NR & NR \\
\hline Serious lung disorder & $\mathrm{AiC}$ & $\mathrm{AiC}$ & $N R$ & $N R$ & NR & NR \\
\hline Chest pain & $\mathrm{AiC}$ & $\mathrm{AiC}$ & $13(7.0)$ & $16(8.3)$ & NR & NR \\
\hline Crackles lung & NR & NR & $13(7.0)$ & 14 (7.3) & NR & NR \\
\hline $\begin{array}{l}\text { Increased upper } \\
\text { airway secretion }\end{array}$ & NR & NR & $12(6.4)$ & $13(6.7)$ & NR & NR \\
\hline
\end{tabular}

Data are presented as $\mathrm{n}$ or $\mathrm{n}(\%)$. DPI: dry powder inhaler; AiC: academic in confidence; NR: not reported. ${ }^{*}$ : number of patients experiencing the event at least once. during the run-in period, patients were normalised to nebulised tobramycin. Patients experiencing adverse events during the run-in period may have withdrawn, or the events may have resolved before randomisation. As such, the population was subject to a degree of selection bias for patients tolerant to nebulised tobramycin. Serious adverse events (which are internationally defined as adverse events that cause death, are life threatening, require hospitalisation/prolong hospitalisation or result in disability or birth defect [22]) appear to occur approximately equally, but slightly less frequently, in the DPI treatments in both the EAGER (DPI 27.4\% versus nebulised 29.2\%) and COLO/DPI/02/06 (DPI 4.3\% versus nebulised $6.2 \%)$ key trials.

In each of the three trials, the percentage of patients withdrawing from the study due to adverse events was higher in the dry powder intervention groups (EAGER 13.0\%; COLO/DPI/02/06 11.8\%; COLO/DPI/02/05 $12.5 \%$ ) than in the nebulised control groups (EAGER 8.1\%; COLO/DPI/02/06 2.6\%; COLO/DPI/02/05 $0 \%)$. Patients in both the EAGER and COLO/DPI/02/06 trials were largely experienced with nebulised tobramycin and, as discussed earlier, it is likely that this difference in dropout rate is at least, in part, due to selection bias of patients tolerant to nebulised tobramycin and desensitisation to its adverse events through prior use.

Table 4 documents the most common adverse events ( $\geqslant 5 \%$ in any group) occurring in any of the three trials. The data presented relate to the number of patients who experienced adverse events. Data on the actual number of events were available for the COLO/DPI/02/06 and COLO/DPI/02/05 studies but are not 
presented here. The most common adverse event in all three trials was cough. The percentage of patients experiencing cough was higher in the COLO/DPI/02/06 and COLO/DPI/02/05 studies than in the EAGER trial, although this may again represent a difference in the definition of cough used in the studies rather than an actual difference in incidence of cough, as the difference persists when comparing the nebulised tobramycin arms of each trial. Cough was more common in the DPI intervention group for all trials. Cough is a known side-effect of dry powder formulations and is thought to generally reduce over time with improved technique, and may be controlled with the use of bronchodilators, to some extent, in some patients.

Whilst no statistical comparisons have been made, other adverse events that appear to be worse in the DPI arm include chest discomfort and dysphonia in the tobramycin DPI arm in the EAGER trial, and throat irritation in the colistimethate sodium DPI arm in the COLO/DPI/02/06 trial. There are minor improvements in a number of some adverse events in the colistimethate sodium DPI arm (table 3 ).

\section{Mortality}

Three patients died in the tobramycin DPI group in the EAGER trial. Two patients died in the tobramycin nebulised group in the COLO/DPI/02/06 trial. None of the deaths are attributed to the study medication, and the studies were not powered for these rare events.

\section{Compliance}

Compliance with study medication was reported in both key trials, but it is not clear whether the definition of compliance, methods used to ascertain compliance and analyses provided are compatible between trials. In the COLO/DPI/02/06 trial, fewer patients were compliant with medication in the colistimethate sodium DPI arm than in the nebulised tobramycin arm $(66.7 \%$ versus $70.7 \%$ respectively complied with $>75 \%$ of doses). The EAGER trial did not define how compliance was judged, but simply states it was generally high with $>90 \%$ compliance in both arms.

\section{Discussion}

Three trials were included in the review of clinical effectiveness. Both colistimethate sodium DPI and tobramycin DPI were reported to be non-inferior to nebulised tobramycin in pivotal phase 3 trials for the outcome FEV1 \%. The smaller trial comparing colistimethate sodium DPI to nebulised colistimethate sodium showed no significant change in lung function in either arm, but was primarily a safety trial. Whilst the key outcome measure in the included trials, $\mathrm{FEV}_{1} \%$, is the standard measure in $\mathrm{CF}$ research, it is considered by some within the research community to be insensitive to small changes, especially in early disease. However, the EMA still recommend that FEV1 \% should be the primary outcome measure, but should be considered in conjunction with microbial outcomes and "harder" outcomes such as acute exacerbations.

The quality of the included studies was generally poor to moderate. None of the trials scored well on all risk of bias items, with issues of blinding and differences in the way that outcomes were defined and measured being key problems. Lack of double-blinding can lead to selection bias and reporting bias for subjective outcomes such as adverse events, inaccuracies and imprecision in the results, and may limit the generalisability of the findings. Failure to blind the outcome assessor (be this the patient, a member of healthcare staff or an independent outcome assessor) can lead to detection bias. This form of bias can lead to differences in how outcomes are determined due to the influence of prior beliefs about the effects of the treatment in question. Therefore, subjectively measured and interpreted data (such as adverse events) should be interpreted with caution.

The data analysis for the EAGER trial included using an ITT analysis without imputation, but failed to provide an analysis at both 20 and 24 weeks. The differences in exclusion of data across the trials in the noimputation, LOCF and completers analyses are likely to affect results, but it is unclear in which direction. The most usual direction of effect of attrition (no imputation and completers analysis) is to overestimate efficacy $[23,24]$. However, LOCF analyses can also have unpredictable results and, in this case, where patients are likely to get progressively worse overtime regardless of treatment, a LOCF analysis may also result in overestimates of efficacy. As such, results from the trials are of unknown comparability and, due to the limitations of the available data, it would not be possible to draw conclusions as to the comparative efficacy of the two dry powder formulations. More patients in the DPI arms withdrew due to adverse events in both trials. The statistical and clinical significance of exacerbation, resistance and adverse event data is not known.

One of the key anticipated benefits of dry powder formulations is an increase in adherence. This claim was not supported by the evidence in this review with lower compliance in the dry powder arm of the COLO/ DPI/02/06 trial and a lack of clarity as to the comparative compliance between arms in the EAGER trial. 
However, there are many issues that may affect compliance and, in the case of the COLO/DPI/02/06 trial, poorer compliance may be due to selection bias as during the run-in period patients took the comparator of nebulised tobramycin; patients who withdrew during this period may have been less satisfied and compliant with the comparator. In the EAGER trial, whilst comparative compliance between nebulised and dry powder formulations was not clear, measurements of patient satisfaction favoured DPI. As such, whilst an increase in compliance has not been demonstrated, clinical experience may reveal a patient preference for dry powder formulations.

We suggest future researchers in this area should prioritise a randomised controlled trial to assess the longer term ( $\geqslant 12$ months) efficacy of colistimethate sodium DPI and tobramycin DPI in comparison to nebulised treatments and in comparison to each other. Future trials should ensure that the EMA guidelines are adhered to, especially in terms of selecting clinically relevant outcomes such as acute exacerbations. Such a study should also include the direct assessment of HRQoL using a relevant preference based instrument to facilitate cost-effectiveness analysis. In addition, high-quality data relating to the relationship between FEV 1 $\%$ and long-term lung health would better inform the argument that FEV $1 \%$ is a useful outcome in trials of short duration. Research conducted by pharmaceutical companies should adhere to recognised medical guidelines such as the EMA and should publish all available results from clinical trials in a timely manner as delayed publication is an area of considerable concern in clinical trials of CF [21].

In summary, colistimethate sodium DPI and tobramycin DPI have both been reported to be non-inferior in terms of FEV1 \% in appropriately powered phase 3 non-inferiority trials at 20 or 24 weeks. However, crucial data on acute exacerbations are not reportedly transparently and are not easily comparable between the trials. A significant number of patients in both trials dropped out from the intervention arms due to adverse events, and cough was reported more often in the DPI treatment groups compared to the nebulised groups. A comparison of colistimethate sodium DPI to tobramycin DPI was not possible due to data limitations and study heterogeneity. Both studies do not adhere to EMA research guidelines and the impact of this on the results is not known. As trials were only 24 weeks in length, the long-term efficacy of either intervention is unknown, and trials recording and powered for non-surrogate outcomes such as exacerbations and mortality over the longer term are required.

\section{Conclusions}

Both DPI formulations have been shown to be non-inferior to nebulised tobramycin as measured by FEV1 \%. However, the results of these trials should be interpreted with caution due to the means by which the results were analysed within the trials, the length of follow-up, and concerns about the ability of FEV $1 \%$ to accurately represent changes in lung health. Clear and comparable data on acute exacerbations, a highly relevant outcome, are missing. However, within trials it would appear that both DPI formulations may increase the likelihood of an exacerbation. It appears that patients on DPI formulations experience more adverse events of cough and may be less likely to tolerate the treatment. As such, the advantages and non-inferiority of DPI treatments compared to nebulised tobramycin remain unclear when all relevant outcomes are considered. Full evaluation of the long-term efficacy of dry powder anti-pseudomonal antibiotics for chronic $P$. aeruginosa is not currently possible and, therefore, phase 4 follow-up trials are required to prove effectiveness for long-term adherence rather than short-term benefit, which will be major driver of drug/device development. Additionally, relevant data on compliance, convenience and HRQoL using a validated measurement are lacking.

\section{Acknowledgements}

The authors would like to thank C. Carroll (School of Health and Related Research (ScHARR), University of Sheffield, Sheffield, UK) who provided support in study selection during the course of the systematic review. The authors also wish to thank Forest Laboratories (Dartford, UK) and Novartis Pharmaceuticals (Camberley, UK) for providing additional data requested throughout the course of the review.

\section{References}

1 European Medicines Agency. Committee for Medicinal Products for Human Use (CHMP) Assessment Report: Colobreathe. EMEA/H/C/001225. 2011.

Cystic Fibrosis Trust. UKCF Registry Annual Data Report. Bromley, Cystic Fibrosis Trust, 2010.

Kosorok MR, Zeng L, West SH, et al. Acceleration of lung disease in children with cystic fibrosis after Pseudomonas aeruginosa acquisition. Pediatr Pulmonol 2001; 32: 277-287.

Cystic Fibrosis Trust. UKCF Registry Annual Data Report. Bromley, Cystic Fibrosis Trust, 2009.

5 Doring G. Prevention of Pseudomonas aeruginosa infection in cystic fibrosis patients. Int J Med Microbiol 2010; 300: 573-577.

Davies JC, Alton EW, Bush A. Cystic fibrosis. BMJ 2007; 335: 1255-1259.

Islam N, Gladki E. Dry powder inhalers (DPIs) - a review of device reliability and innovation. Int J Pharm 2008; 360: 1-11. 
National Institute for Health and Clinical Excellence (NICE). Colistimethate sodium and tobramycin dry powders for inhalation for treating pseudomonas lung infection in cystic fibrosis. 2010. www.nice.org.uk/nicemedia/live/ 14134/63269/63269.pdf Date last updated: May 2013. Date last accessed: September 30, 2013.

9 Tappenden P, Harnan S, Uttley L, et al. Colistimethate sodium powder and tobramycin powder for inhalation for the treatment of Pseudomonas aeruginosa lung infection in cystic fibrosis [ID342]. 2012. www.nice.org.uk/ nicemedia/live/13068/61112/61112.pdf Date last updated: March 13, 2012. Date last accessed: September, $30: 2013$.

10 European Medicines Committee. Committee for Medicinal Products for Human Use (CHMP). Guideline on the clinical development of medicinal products for the treatment of cystic fibrosis. 2009. www.ema.europa.eu/docs/ en_GB/document_library/Scientific_guideline/2009/12/WC500017055.pdf Date last updated: October 22, 2009. Date last accessed: September 30, 2013.

11 Centre for Reviews and Dissemination. Systematic Reviews. CRD's guidance for undertaking systematic reviews in health care. York, University of York, 2009.

12 Moher D, Liberati A, Tetzlaff J, et al. Preferred reporting items for systematic reviews and meta-analyses: the PRISMA statement. PLoS Med 2009; 6: e1000097.

13 Konstan MW, Flume PA, Kappler M, et al. Safety, efficacy and convenience of tobramycin inhalation powder in cystic fibrosis patients: the EAGER trial. J Cyst Fibros 2011; 10: 54-61.

14 Davies JC, Hall P, Francis J, et al. A dry powder formulation of colistimethate sodium is safe and well tolerated in adults and children with CF. Pediatr Pulmon 2004; 38: Suppl. 27, 283

15 Konstan M, Flume PA, Brockhaus G, et al. Safety and efficacy of tobramycin inhalation powder (TIP) in treating CF patients infected with Pseudomonas aeruginosa (Pa). J Cyst Fibros 2010; 9: Suppl. 1, S22.

16 Novartis Pharmaceuticals. Colistimethate sodium powder (Colobreathe ${ }^{\circledR}$ ) and tobramycin powder for inhalation (TOBI Podhaler ${ }^{\circledR}$ ) for the treatment of pseudomonas lung infection in cystic fibrosis - Novartis submission to NICE. Novartis submission to NICE 2011. www.nice.org.uk/nicemedia/live/13068/61134/61134.pdf Date last accessed: September 30, 2013.

17 Forest Laboratories UK Ltd. Colistimethate sodium powder for inhalation for the treatment of Pseudomonas lung infection in cystic fibrosis - Forest Submission to NICE. Forest Submission to NICE 2011. www.nice.org.uk/ nicemedia/live/13068/61132/61132.pdf Date last accessed: September 30, 2013

18 European Medicines Agency. Committee for Medicinal Products for Human Use (CHMP). Assessment Report: TOBI Podhaler. EMEA/H/C/002155. London, ECDC, 2012.

19 Schuster A, Haliburn C, Döring G, et al. Safety, efficacy and convenience of colistimethate sodium dry powder for inhalation (Colobreathe DPI) in patients with cystic fibrosis: a randomised study. Thorax 2013; 68: 344-350.

20 Ramsey BW, Pepe MS, Quan JM, et al. Intermittent administration of inhaled tobramycin in patients with cystic fibrosis. Cystic Fibrosis Inhaled Tobramycin Study Group. N Engl J Med 1999; 340: 23-30.

21 Hurley MN, Prayle AP, Smyth AR. Delayed publication of clinical trials in cystic fibrosis. J Cyst Fibros 2012; 11: $14-17$.

22 International conference on harmonisation of technical requirements for registration of pharmaceuticals for human use. ICH harmonised tripartite guideline: Clinical safety data management - definitions and standards for expedited reporting E2A. 1994. www.ich.org/fileadmin/Public_Web_Site/ICH_Products/Guidelines/Efficacy/E2A/ Step4/E2A_Guideline.pdf Date last updated: October 27, 1994. Date last accessed: September 30, 2013.

23 Terzano C, Taurino AE, Peona V. Nebulized tobramycin in patients with chronic respiratory infections during clinical evolution of Wegener's granulomatosis. Eur Rev Med Pharmacol Sci 2001; 5: 131-138.

24 Robinson CA, Kuhn RJ, Craigmyle J, et al. Susceptibility of Pseudomonas aeruginosa to cefepime versus ceftazidime in patients with cystic fibrosis. Pharmacotherapy 2001; 21: 1320-1324. 